\title{
Measurement of Moisture Diffusion as a Function of Temperature and Moisture Concentration in Transformer Pressboard
}

\author{
Y. Du, A. V. Mamishev, B. C. Lesieutre, and M. Zahn \\ Massachusetts Institute of Technology \\ Department of Electrical Engineering and Computer Science \\ Laboratory for Electromagnetic and Electronic Systems \\ Cambridge, MA 02139, U.S.A.
}

\begin{abstract}
The moisture diffusion process is measured for oilfree transformer pressboard using a flexible interdigital dielectrometry three-wavelength sensor. Experiments are performed for five different temperatures at various moisture levels. The diffusion coefficient as a function of temperature and moisture concentration is estimated and compared with literature values. The numerical methods of solving the diffusion equation are discussed.

\section{Introduction}

Interdigital frequency-wavenumber dielectrometry is based on the excitation of several sets of spatially periodic interdigitated electrodes with a sinusoidal voltage frequency sweep. Preliminary measurements that demonstrate the response of three-wavelength $(1.0,2.5$, and $5.0 \mathrm{~mm}$ ) sensor to the molecular diffusion process were presented in [1]. Similar tests using interdigital sensor have been done by other investigators to measure moisture diffusion in thin epoxy films [2].
\end{abstract}

A companion paper [3] describes the details of the sensor design and measurement setup. It uses the output of three wavelengths at $70^{\circ} \mathrm{C}$ to find the moisture spatial profiles and then simulates the diffusion process using a constant moisture diffusion coefficient model until the numerical model reasonably matches the experimental profile. In this paper, a linear relation between the transcapacitance and the moisture concentration is also assumed, and the output of the shortest wavelength of 1 $\mathrm{mm}$ at different temperatures is used to find the diffusion coefficient as a function of concentration and temperature. The shortest wavelength provides the most accurate values of moisture concentration because the moisture distribution near the zero flux boundary is essentially constant within its relatively small penetration depth.

\section{Experiment}

The interdigital sensor is attached to one side of the pressboard, whereas the other side of the pressboard is exposed to ambient conditions through a perforated steel plate. The four side edges of the pressboard sample are sealed with silicon glue to only allow one dimensional diffusion transverse to the pressboard surface. The test sandwich structure is placed in a vacuum-tight chamber whose temperature and moisture can be monitored and controlled [1].

The tests were performed at five temperatures: 30,40 , 50,60 , and $70^{\circ} \mathrm{C}$. Hi-Val pressboard, manufactured by EHV-Weidmann Industries, Inc., is used in all experiments. The samples are $1.5 \mathrm{~mm}$ thick except for measurements at $30^{\circ} \mathrm{C}$ where the pressboard thickness is $1.0 \mathrm{~mm}$. The reason for this exception is that the diffusion process is very long at low temperatures due to a small diffusion coefficient. The reduction of thickness from $1.5 \mathrm{~mm}$ to $1.0 \mathrm{~mm}$ shortens the diffusion time by approximately $56 \%$. At each constant temperature, the pressboard was first vacuum-dried for over 24 hours. This gives a uniform zero initial moisture distribution. The moisture diffusion process starts by bubbling a constant flow of moist air.

This paper analyzes the signals from the sensing electrodes taken at $1 \mathrm{~Hz}$ sinusoidal frequency. The $1 \mathrm{~mm}$ wavelength sensor measures the transimpedance of the region close to the sensor-pressboard interface. The moisture concentration at the sensor-pressboard interface at any time during the diffusion process is measured using a linear relationship between the transcapacitance and the moisture concentration.

\section{Boundary Condition}

The measurement schematic is shown in Figure 1. The moist air flow keeps the relative humidity of the testing chamber at a nearly constant level, which gives a constant moisture boundary condition at the pressboard-air interface at $x=l$. The other side of the pressboard is against the sensor at $x=0$, which imposes a zero flux boundary condition. 


\begin{tabular}{|l|c|c|c|c|c|}
\hline \hline $\mathbf{T}\left({ }^{\circ} \mathbf{C}\right)$ & $\mathbf{3 0}$ & $\mathbf{4 0}$ & $\mathbf{5 0}$ & $\mathbf{6 0}$ & $\mathbf{7 0}$ \\
\hline$a_{6}\left(\times 10^{-11}\right)$ & -9.3468655 & -3.8729882 & 9.5319144 & 7.4380470 & 16.252499 \\
\hline$a_{5}\left(\times 10^{-8}\right)$ & 4.2446633 & 2.5228351 & -1.1755019 & -0.75286519 & -3.3218692 \\
\hline$a_{4}\left(\times 10^{-6}\right)$ & -6.3395879 & -4.2926236 & -0.37397578 & -0.57129397 & 2.2473555 \\
\hline$a_{3}\left(\times 10^{-4}\right)$ & 4.3831525 & 3.2448905 & 1.2731316 & 1.1731076 & -0.27101029 \\
\hline$a_{2}\left(\times 10^{-3}\right)$ & -14.929696 & -11.950117 & -7.2850779 & -6.2300223 & -2.7797731 \\
\hline$a_{1}\left(\times 10^{-1}\right)$ & 3.2828657 & 2.9079147 & 2.4316118 & 2.1359436 & 1.7762623 \\
\hline$a_{0}\left(\times 10^{-1}\right)$ & 2.4131270 & 1.6954583 & 1.0483257 & 1.3978572 & 0.7441865 \\
\hline \hline
\end{tabular}

Table 1: Polynomial fitting parameters for partition curves for ambient air relative humidity and moisture in paper for various temperatures.

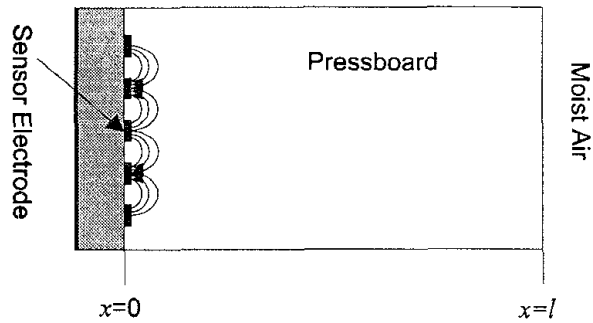

Figure 1: $1 \mathrm{~mm}$ wavelength sensor detects changes at the pressboard-sensor surface at $x=0$ while the moisture diffuses from the ambient at $x=l$.

The air relative humidity is monitored by a commercial relative humidity sensor and the value is converted to the moisture in pressboard at the boundary using airpressboard equilibrium curves drawn by Oommen [4] from Jeffries' data [5]. At each temperature each curve is fitted with a 6 th-order polynomial to be conveniently used in the algorithm

$$
y=a_{6} x^{6}+a_{5} x^{5}+a_{4} x^{4}+a_{3} x^{3}+a_{2} x^{2}+a_{1} x+a_{0},
$$

where $x$ is the air relative humidity in percent and $y$ is the moisture in paper in percent by weight. The coefficients in (1) are shown in Table 1 . The moisture equilibrium curves reconstructed from the 6-th order polynomial are shown in Figure 2.

\section{Diffusion Analysis}

Our goal is to find the diffusion coefficient $D$ and the moisture spatial profiles during the entire diffusion process. The diffusion of moisture is defined mathematically in one dimension by Fick's second law of diffusion:

$$
\frac{\partial C}{\partial t}=\frac{\partial}{\partial x}\left(D \frac{\partial C}{\partial x}\right) \text {. }
$$

When $D$ is a constant, closed form solution for zero initial distribution and constant boundary condition $C_{1}$ at $x=l$ and zero flux at $x=0$ is

$C=C_{1}\left\{1-\frac{4}{\pi} \sum_{n=0}^{\infty} \frac{(-1)^{n}}{2 n+1} e^{-t / \tau_{n}} \cos \frac{(2 n+1) \pi x}{2 l}\right\}$

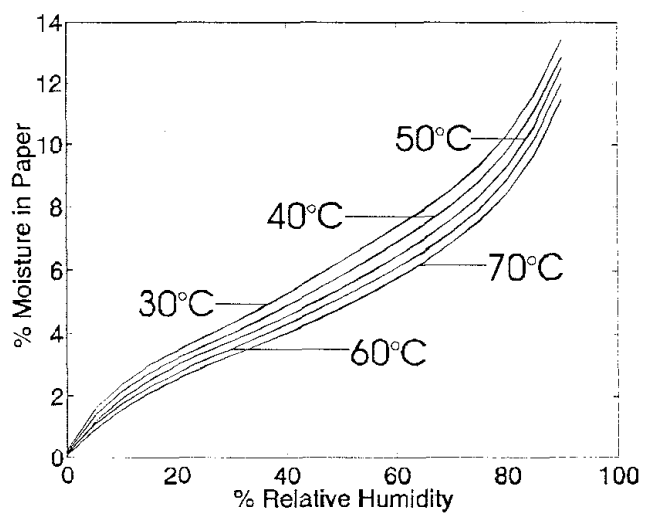

Figure 2: Moisture in wood pulp as a function of ambient relative humidity curves reconstructed from the 6-th order polynomial fit to Jeffries'[5] data.

where

$$
\tau_{n}=\frac{4 l^{2}}{D(2 n+1)^{2} \pi^{2}} .
$$

When the diffusion coefficient is concentrationdependent, equation (2) becomes non-linear and no general analytical solution is available. Numerical methods are then employed to solve the diffusion equation.

In 1974 Guidi and Fullerton in their paper [6] gave an empirical fit for diffusion coefficients of oil-impregnated Kraft paper from data studied by Ewart:

$$
D(C, T)=D_{0} e^{k C+E_{\iota t}\left(1 / T_{0}-1 / T\right)}
$$

where $D_{0}=6.44 \times 10^{-14} \mathrm{~m}^{2} / \mathrm{s}, k=0.5, C$ is the moisture concentration in weight percent per unit weight dry cellulose, $E_{a}=7700^{\circ} \mathrm{K}$, and $T$ is the absolute Kelvin temperature. They didn't specify $T_{0}$; however, from Foss's work in 1987 [7], it appears that $T_{0}=$ $298^{\circ} \mathrm{K}$

Foss [7] generalized the empirical work at General Electric and fitted the diffusion coefficient for both oil-free and oil-impregnated paper into the same form as Guidi and Fullerton in (5) with $T_{0}=298^{\circ} \mathrm{K}$ and $k=0.5$. The corresponding parameters are shown in Table 2. 


\begin{tabular}{|l|c|c|}
\hline & $D_{0}$ & $E_{a}$ \\
\hline Oil-free & $2.62 \times 10^{-11} \mathrm{~m}^{2} / \mathrm{s}$ & $8140^{\circ} \mathrm{K}$ \\
\hline Oil-impregnated & $1.34 \times 10^{-13} \mathrm{~m}^{2} / \mathrm{s}$ & $8074^{\circ} \mathrm{K}$ \\
\hline
\end{tabular}

Table 2: Diffusion coefficient parameters estimated by Foss [7].

\begin{tabular}{|c|c|c|}
\hline \hline$D_{0}$ & $\mathrm{k}$ & $\overline{E_{a}}$ \\
\hline $0.67 \times 10^{-12} \mathrm{~m}^{2} / \mathrm{s}$ & 0.45 & $7646^{\circ} \mathrm{K}$ \\
\hline
\end{tabular}

Table 3: Diffusion coefficient parameters obtained from measurements using a least square fit.

The same temperature and moisture concentration dependence model of (5) is used here to find the corresponding parameters, $D_{0}, k$, and $E_{a}$, for Hi-Val pressboard from our experiments.

\section{Let}

$$
d z=D(C) d C,
$$

then (2) becomes

$$
\frac{\partial C}{\partial t}=\frac{\partial^{2} z}{\partial x^{2}}
$$

Equation (7) is discretized for numerical calculation

$$
\frac{C_{j}^{n+1}-C_{j}^{n}}{\Delta t}=\frac{z_{j+1}^{n+1}-2 z_{j}^{n+1}+z_{j-1}^{n+1}}{(\Delta x)^{2}}
$$

where superscript $n$ corresponds to time steps and subscript $j$ corresponds to space steps. This backward time scheme is chosen for stability of the algorithm [8].

Then, $z$ can be derived in terms of $C$ from

$$
d z=D(C) d C=D_{0} e^{k C+E_{a}\left(1 / T_{0}-1 / T\right)} d C,
$$

as

$$
z=\frac{D_{0}}{k} e^{k C+E_{a l}\left(1 / T_{0}-1 / T\right)} .
$$

Standard numerical procedures are then carried out to solve (8).

To find the parameters for the diffusion coefficient, the measured moisture concentration by the $1 \mathrm{~mm}$ wavelength sensor is taken at five different temperatures and the Matlab built-in function for least square fit leastsq in the optimization toolbox is used to find the parameters that minimize the sum of the error squares of the moisture concentration. The results are shown in Table 3.

\section{Discussion}

For $T=70^{\circ} \mathrm{C}$, the diffusion coefficient at the start when $C=0 \%$ is $1.9 \times 10^{-11} \mathrm{~m}^{2} / \mathrm{s}$, and at the end when $C=$

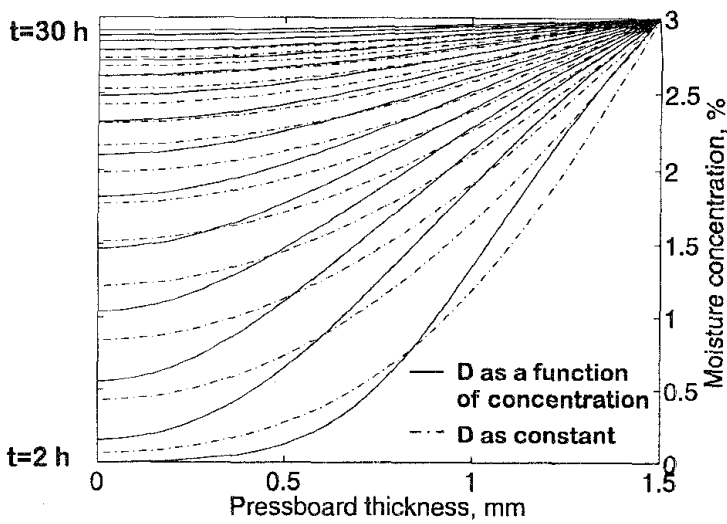

Figure 3: Calculated moisture profile in the pressboard at $60^{\circ} \mathrm{C}$ for diffusion coefficient as a function of concentration using estimated parameters and as a constant $D=2.4 \times 10^{-11} \mathrm{~m}^{2} / \mathrm{s}$, an average of $D$ at $C=0 \%$ and $C=3.0 \%$.

$1.8 \%$ is $4.3 \times 10^{-11} \mathrm{~m}^{2} / \mathrm{s}$. The average is about $3.1 \times$ $10^{-11} \mathrm{~m}^{2} / \mathrm{s}$. This is close to the value that is estimated in [3] for a constant diffusion coefficient model, $2.3 \times$ $10^{-11} \mathrm{~m}^{2} / \mathrm{s}$. In this paper, the diffusion coefficient is found by minimizing the sum of the error squares between the measured moisture concentration and the theoretical values for various temperatures, whereas the approach in [3] uses a constant diffusion coefficient to do forward simulation until the calculated profile closely matches most of the measured profile.

The spatial profiles of the diffusion process at $60^{\circ} \mathrm{C}$ with the diffusion coefficient as a function of concentration, and as a constant $D=2.4 \times 10^{-11} \mathrm{~m}^{2} / \mathrm{s}$ (an average of $D$ at $C=0 \%$ and $C=3.0 \%$ ) are shown in Figure 3. The curves are plotted from 2 hours to 30 hours at 2-hour intervals. At first, since the moisture concentration is low, the diffusion coefficient in most regions is smaller than the average value, the curves for constant diffusion coefficient increase faster than the concentration dependent model. When the moisture in the pressboard increases, the diffusion speed also increases, thus the concentration dependent curves exceed the constant diffusion curves. This also explains why the experimental data in Figure 5 of [3] shows that the moisture reaches equilibrium in a time of 28 hours, whereas the constant diffusion coefficient simulation in Figure 6 of [3] has not yet reached equilibrium after 28 hours.

The diffusion coefficient for oil-free pressboard calculated here is much smaller than that reported in [7]. This could be due to three reasons. First, the materials are different. Foss' data are for A50P281A Kraft paper 


\begin{tabular}{|l|c|c|c|}
\hline \hline Source & MIT & Foss [7] & Quarshie [9] \\
\hline Material & Pressboard & Kraft paper & Manilla paper \\
\hline$C=2.1 \%$ & $2.5 \times 10^{-11}$ & $1.3 \times 10^{-9}$ & $1.3 \times 10^{-11}$ \\
\hline$C=3.0 \%$ & $3.8 \times 10^{-11}$ & $2.1 \times 10^{-9}$ & $4.8 \times 10^{-11}$ \\
\hline \hline
\end{tabular}

Table 4: A comparison of our measured diffusion coefficient $\left(\mathrm{m}^{2} / \mathrm{s}\right)$ and literature reported values at $60^{\circ} \mathrm{C}$.

made from $100 \%$ sulphate fiber with thickness of 0.01 inch. The reported value in [9] by Quarshie is close to our results given in Table 4. Quarshie used 50 layers of Manilla paper each $0.045 \mathrm{~mm}$ thick.

Second, our sample is tightly compressed. Pressure has been shown to slow down the diffusion process [9]. For the EHV Weidmann T-IV pressboard that Quarshie tested, the diffusion coefficient of unclamped pressboard is about 1.3 times that of clamped pressboard.

Third, due to the porous squeezing structure at the pressboard-air interface, the pressboard is not completely exposed to the ambient at that interface. This might slow down the diffusion, but the effect should be small because the diffusion in the direction parallel to the interface is much faster than that of the direction perpendicular to the interface which we are studying.

\section{Conclusions}

The diffusion process in oil-free pressboard is monitored at five different temperatures using interdigital dielectrometry sensor technology. An algorithm is developed for finding the diffusion coefficient as a function of moisture concentration and temperature.

Future experiments will combine a transformer oil convective flow facility and the interdigital sensor setup for studying temperature and moisture transients in oilimpregnated pressboard. That facility will minimize the second and third effects discussed above. Other insulating materials would be tested and compared with literature values.

\section{Acknowledgments}

The authors would like to gratefully acknowledge the financial support of the Electric Power Research Institute, under grant WO 8619-01, managed by Mr. S. Lindgren, the National Science Foundation under grant No. ECS-9523128, the Demonstration of Energy-Efficiency Developments Scholarship from American Public Power Association, sponsored by Belmont Utility managed by Mr. T. McCarthy, and Link Foundation Fellowship. The authors would like to thank Mr. Wayne Ryan of MIT for assistance with experiments.

\section{References}

[1] Y. Du, B. Lesieutre, and M. Zahn, "Dielectrometry measurements of effects of moisture and anti-static additive on transformer board," in IEEE Conference on Electrical Insulation and Dielectric Phenomena, (Minneapolis, MN), pp. 226-229, Oct. 1997.

[2] D. R. Day, D. D. Shepard, and K. J. Craven, "A microdielectric analysis of moisture diffusion in thin epoxy/amine films of varying cure state and mix ratio," Polymer Engineering and Scinece, vol. 32, pp. 524-528, Apr. 1992.

[3] A. V. Mamishev, Y. Du, B. C. Lesieutre, and M. Zahn, "Measurement of moisture spatial profiles in transformer pressboard," in IEEE Conference on Electrical Insulation and Dielectric Phenomena, (Atlanta, GA), Oct. 1998.

[4] T. V. Oommen, "Moisture equilibrium in paperoil insulation systems," in Proceedings of the 16 th Electrical/Electronics Insulation Conference, (Chicago, IL), pp. 162-166, Oct. 3-6, 1983.

[5] R. Jeffries, "The sorption of water by cellulose and eight other textile polymers," Journal of the Textile Institute Transactions, vol. 51, no. 9, pp. 339-374, 1960.

[6] W. W. Guidi and H. P. Fullerton, "Mathematical methods for prediction of moisture take-up and removal in large power transformers," Proceedings of IEEE Winter Power Meeting, no. C-74, pp. 242244, 1974.

[7] S. Foss, "Power transformer drying model," Tech. Rep. DS-002-87, Dynamic Systems, Pittsfield, MA, Oct. 1987. Prepared for General Electric Company, Large Transformer Operation, Pittsfield, MA, and Consolidated Edison Corporation, New York, NY.

[8] W. Press, S. Teukolsky, W. Vetterling, and B. Flannery, Numerical Recipes in C: The Art of Scientific Computing, ch. 19. Cambridge: Cambridge University Press, 1992.

[9] J. Quarshie, "Diffusion of moisture through cellulose insulation," Master's thesis, Department of Electrical and Electronic Engineering, University of Nottingham, Oct. 1977. 